\title{
Control of EV Charging Points for Thermal and Voltage Management of LV Networks
}

\author{
Jairo Quirós-Tortós, Member, IEEE, Luis F. Ochoa, Senior Member, IEEE, \\ Sahban W. Alnaser, Member, IEEE, and Tim Butler
}

\begin{abstract}
High penetrations of domestic electric vehicles (EVs) in UK low voltage (LV) networks may result in significant technical problems. This paper proposes an implementable, centralized control algorithm, currently being trialed in $9 \mathrm{UK}$ residential LV networks, that uses limited information to manage EV charging points to mitigate these technical problems. Two real UK LV networks are used to quantify the potential impacts of different EV penetration levels and to demonstrate the effectiveness of the control algorithm (using different control cycles) for simultaneous thermal and voltage management. Monte Carlo simulations (adopting 1-min resolution data) are undertaken to cater for domestic and EV demand uncertainties. Results for these LV networks show that problems may occur for $\mathrm{EV}$ penetrations higher than $20 \%$. More importantly, they highlight that even for a $100 \%$ penetration and control cycles of up to $10 \mathrm{~min}$, the control algorithm successfully mitigates problems on the examined LV networks. Crucially, to determine effects on the comfort of EV users, a metric is introduced and discussed. The results of different control settings are presented to analyze potential adaptations of the control strategy. Finally, a comparison with an optimization framework highlights that the proposed algorithm is as effective whilst using limited information.
\end{abstract}

Index Terms--Electric vehicles, low voltage networks, thermal management, voltage management, real-time control.

\section{INTRODUCTION}

$\mathrm{T}$ HE adoption of domestic electric vehicles (EVs) in the UK is expected to increase in the near future given their contribution in reducing greenhouse gases and dependency on fossil fuels [1]. However, the uncontrolled charging of EVs may result in significant technical problems on the residential low voltage (LV) electricity networks (e.g., thermal problems on transformers/cables and/or significant voltage drops) [2, 3].

To cope with the potential impacts of EVs on distribution networks, different EV charging strategies have been suggested [4-13]. These studies propose the use of complex optimization techniques (e.g., linear [4-6], non-linear [7], dynamic [8], quadratic [9, 10], mix-integer linear programming [11], and receding-horizon [12]) that require extensive information/ visibility of the network (e.g., voltages and currents); the state of charge (SOC) of the EVs; and, typically, the electricity market (e.g., real-time pricing). In practice, however, real-time data is limited and many interoperability challenges (e.g., data

This work has been funded by EA Technology Limited, UK, through the Ofgem's Low Carbon Networks Fund Tier 2 Project "My Electric Avenue", 2013-2015. J. Quirós-Tortós, L. F. Ochoa and S.W. Alnaser are with the School of Electrical and Electronic Engineering, The University of Manchester, UK. T. Butler is with EA Technology Limited, UK. (e-mails: jairoquirostortos@ieee.org, luis_ochoa@ieee.org, sahban.alnaser@manchester.ac.uk, timothy.butler@eatechnology.com). exchanges between EV and charging point) need to be addressed [14]. Thus, the data and infrastructure requirements of these approaches make their adoption by Distribution Network Operators (DNOs) unfeasible in the short and mid-terms.

In addition, most of these studies are focused on mitigating the aggregated EV impacts on medium voltage (MV) circuits (i.e., >1 kV) [6-11]. However, highly dense residential LV networks, such as those in Europe (multiple feeders, hundreds of customers), are likely to be the first bottleneck and thus the corresponding impacts must be investigated. For this, the adoption of real LV networks is critical given that simplified models (e.g., [5]) may not reveal their particularities [4, 15].

From the customer perspective, it is also important to ensure that expectations of comfort are met [16]. Hence, not only the LV network capacity and voltage constraints must be satisfied, but it is also critical to quantify the effects that any EV charging strategy has on the expected charging time/level of EVs. This, however, has not been assessed in the above works, which are limited to price aspects only $[6,7,11,12]$.

This paper proposes an implementable, centralized control algorithm that manages EV charging points to mitigate technical problems (i.e., thermal/voltage) in LV networks. This is done by adopting corrective and preventive approaches for the disconnections and reconnections of EV charging points, respectively. The conceptual strategy is based on the Esprit Technology (patent PCT/GB2012/050702 [17]) and is currently being trialed in $9 \mathrm{UK}$ residential LV networks involving 86 EVs [18]. The infrastructure required by the proposed control strategy (and deployed in the trial) includes controllable EV charging points, communication links, substation sensors, and a substation PLC-based controller. This makes the approach very practical, and thus, more attractive to DNOs as a solution towards the transition to complex Smart Grid schemes.

Two real LV networks in the North West of England are used to quantify the impacts of different EV penetrations and to demonstrate the effectiveness of the control algorithm for simultaneous thermal and voltage management. The impacts of uncontrolled and controlled EV charging are presented in terms of asset utilization and number of customers with voltage problems. To cater for the uncertainties related to household demand as well as EV demand and location, the analyses are carried out using a Monte Carlo approach [15, 19] considering 1-min resolution data and unbalanced (three-phase) LV network models with single-phase customer connection points.

Crucially, the effects that the proposed control algorithm has on customers are assessed and discussed through a new impact metric that quantifies the delay in the controlled charg-

ing time compared to the uncontrolled charging time. The number of control actions (i.e., disconnections/reconnections) 


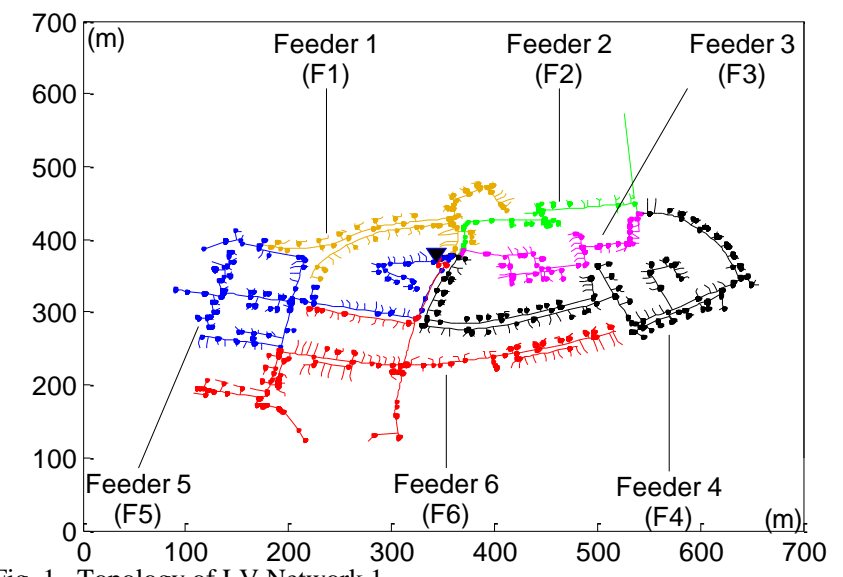

Fig. 1. Topology of LV Network 1

TABLE I

MAIN ELECTRICAL CHARACTERISTICS OF LV NETWORK 1

\begin{tabular}{ccccccc}
\hline \hline & $\mathrm{F} 1$ & $\mathrm{~F} 2$ & $\mathrm{~F} 3$ & $\mathrm{~F} 4$ & $\mathrm{~F} 5$ & $\mathrm{~F} 6$ \\
\hline \# Customers & 49 & 21 & 30 & 100 & 68 & 83 \\
Phase A $(\%)$ & 40.8 & 42.9 & 36.6 & 30.6 & 42.9 & 38.7 \\
Phase B $(\%)$ & 32.7 & 23.8 & 26.8 & 34.7 & 26.5 & 28.6 \\
Phase C $(\%)$ & 26.5 & 33.3 & 36.6 & 34.7 & 30.6 & 32.7 \\
Length $(\mathrm{m})$ & 272.8 & 374.3 & 270.9 & 640.3 & 414.0 & 431.3 \\
$R(\Omega / \mathrm{km})$ & 27.8 & 17.6 & 17.7 & 17.6 & 31.9 & 33.4 \\
$X(\Omega / \mathrm{km})$ & 6.6 & 4.3 & 6.4 & 7.3 & 5.5 & 6.3 \\
\hline \hline
\end{tabular}

is also quantified to assess the potential effects on EV battery. The results of different control settings are also presented to analyze potential adaptations of the control algorithm. Finally, to understand the extent to which the proposed approach provides effective network and EV management, a comparison with an ideal optimization framework is carried out.

This paper is structured as follows. Section II describes the input data used in this work. Section III presents a probabilistic EV impact assessment showing the importance of the hierarchical control algorithm formally introduced in section IV. Section V demonstrates the effectiveness of the control algorithm on two real UK LV networks and presents potential adaptations. The results of the control algorithm are compared against an ideal optimal solution in section VI. A discussion is provided in section VII and conclusions are drawn in section VIII. Real data from the trial is presented in the appendix.

\section{II.INPUT DATA: LV NETWORKS AND LOAD PROFILES}

This section describes the input data used in this work and summarizes the Monte Carlo approach used to quantify the impacts of uncontrolled and controlled EV charging.

\section{A. LV Networks}

Two real LV networks from the North West of England are used in this work. These UK LV networks, with different customer numbers and feeder lengths, are fully modeled using OpenDSS [20]. The topologies and main characteristics (e.g., the length and average impedance of the main path) are shown in Fig. 1 and Table 1, and Fig. 2 and Table 2. It is important to mention that all the LV feeders are operated radially by using open points. Moreover, the LV feeders are three-phase with single-phase customer connection points. The distribution of customers among the phases in these residential LV networks is shown in Table I and Table II.

LV Network 1 (Fig. 1, Table 1) consists of six feeders and 351 customers. LV Network 2 (Fig. 2, Table 2) consists of

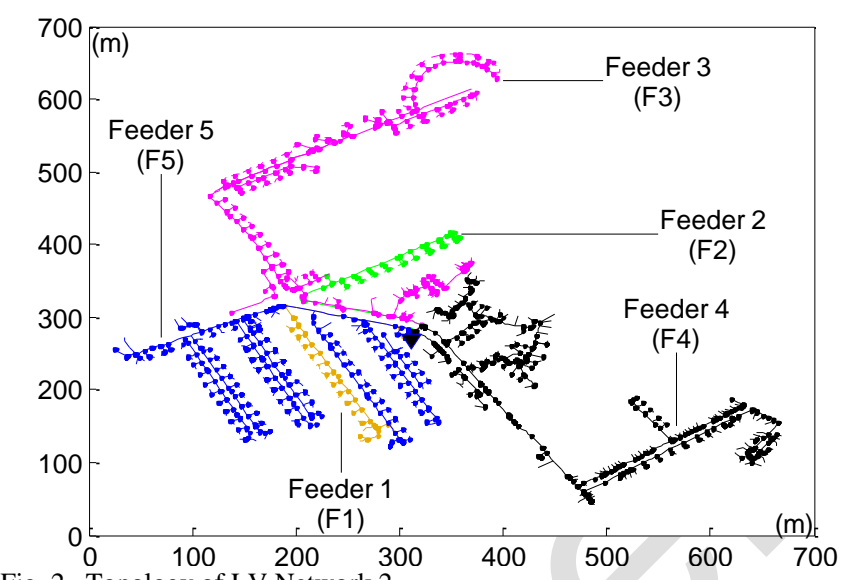

Fig. 2. Topology of LV Network 2

TABLE II

MAIN ELECTRICAL CHARACTERISTICS OF LV NETWORK 2

\begin{tabular}{cccccc}
\hline \hline & $\mathrm{F} 1$ & $\mathrm{~F} 2$ & $\mathrm{~F} 3$ & $\mathrm{~F} 4$ & $\mathrm{~F} 5$ \\
\hline \# Customers & 23 & 15 & 106 & 135 & 149 \\
Phase A $(\%)$ & 39.1 & 26.7 & 36.7 & 28.6 & 42.9 \\
Phase B $(\%)$ & 34.8 & 33.3 & 32.7 & 42.8 & 30.6 \\
Phase C $(\%)$ & 26.1 & 40.0 & 30.6 & 28.6 & 26.5 \\
Length $(\mathrm{m})$ & 371.4 & 341.9 & 721.0 & 655.0 & 421.0 \\
$R(\Omega / \mathrm{km})$ & 15.0 & 23.4 & 24.5 & 32.8 & 27.7 \\
$X(\Omega / \mathrm{km})$ & 4.4 & 3.7 & 6.0 & 7.3 & 6.8 \\
\hline \hline
\end{tabular}

five feeders and 428 customers. The networks are supplied by three-phase 500 and $800 \mathrm{kVA}$ single distribution transformers, (black triangles), respectively. The busbar voltage of the transformers is $416 \mathrm{~V}$ (line-to-line) as per UK DNO practice.

\section{B. Domestic Load Profiles}

All the LV network customers are modeled as domestic unrestricted [21], i.e., heating needs are primarily supplied by gas. The time-series behavior (considering $1 \mathrm{~min}$ resolution data) of the domestic loads is created using the CREST tool [22] - a tool that considers the domestic behavior of UK costumers, the number of people at home, the type of day, the month, and the use of the appliances. The domestic load profiles used in this work correspond to a typical weekday during winter (e.g., January), i.e., maximum demand in the UK. To mimic the stochastic behavior of the load consumption per household, a pool of 1000 different load profiles was created. This pool considers the proportion of houses with one, two, three and four or more people: $29,35,16$ and 20\%, respectively, based on the UK National Statistics [23]. To illustrate this, Fig. 3(a) presents three residential load profiles and Fig. 3(c) shows the diversified demand for the 1000 load profiles.

\section{EV Load Profiles}

The time-series behavior ( 1 min resolution) of the EVs is obtained using the stochastic data reported in [3] (start charging time and energy demanded during a connection), as the result of a one-year EV trial in Ireland. The probabilities of the start charging time are shifted three hours earlier to create a higher coincidence with the peak demand in the UK $(\sim 7 \mathrm{pm})$. This further stresses the LV network during peak hours; thus the benefits of the control algorithm are more evident. These probabilities are used to create a pool of $1000 \mathrm{EV}$ profiles. Fig. 3(b) presents three EV profile examples and Fig. 3(c) shows the diversified demand for the $1000 \mathrm{EV}$ profiles.

When not managed, the EV charging process is assumed to be continuous, i.e., once it starts, it will not end until the bat- 


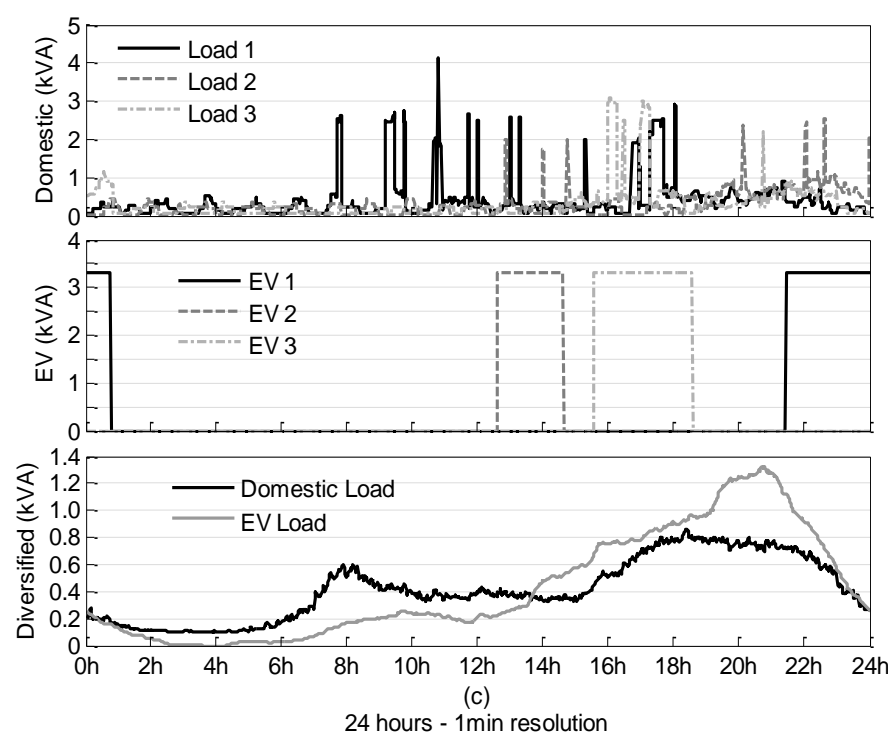

Fig. 3. Domestic and EV load profiles: (a) Individual load profiles (b) Individual EV profiles (c) Diversified demand for 1000 load and EV profiles

tery stops withdrawing power (e.g., the EV is disconnected or fully charged). It is considered that EVs are connected only once per day, and their state of charge (SOC) is not known. The EVs utilized are similar to the commercially available Nissan LEAF, i.e., the battery capacity is $24 \mathrm{kWh}$ [24]. As EVs are connected at home, only the slow charging mode is considered, i.e., a constant charging rate of $3.3 \mathrm{~kW}$ is used.

\section{D.Monte Carlo-based Methodology and Impact Metrics}

The Monte Carlo-based methodology developed in [15, 19] is used to quantify the impacts of uncontrolled and controlled EV charging considering 100 simulations. This caters for the uncertainties related to household demand as well as EV demand and location. At each simulation, a randomly selected domestic load profile from the pool is allocated to each customer node to mimic its demand. Similarly, an EV load profile is randomly allocated at customer nodes according to the penetration level. A given penetration indicates the percentage of houses with an EV. There is a maximum of one EV per household irrespective of the number of residents. Moreover, uneven penetrations per feeder are allowed. The EV penetration is increased from 0 (base case) to $100 \%$ in steps of $10 \%$. When load profiles are allocated to customer nodes, a timeseries three-phase power flow is solved using OpenDSS.

For each Monte Carlo simulation, the next indices (performance metrics) are calculated to quantify the EV impacts:

- Transformer $(T x)$ utilization factor: Calculated as the hourly maximum three-phase power through the transformer divided by its capacity. The three-phase power is obtained by averaging the results from the 1-min power flow simulations within each moving hour. This 1 -hour period takes into account the thermal time constant of the assets $[25,26]$.

- Feeder utilization factor: Calculated as the hourly maximum phase current through the first segment (head) of the feeder divided by its corresponding capacity. The phase current is obtained by averaging the results from the 1-min power flow simulations within each moving hour.

- Costumers with voltage problems: It checks whether the voltages calculated at the single-phase connection point of each costumer from the time-series power flow simulations satisfy the UK voltage regulation of $+10 \% /-6 \%$ for $95 \%$ of the time [27]. This standard, which considers 10-min averages during a week, is limited to only one day.

\section{EV IMPACTS ON LV NETWORKS}

This section illustrates the extent and diversity of technical problems (i.e., thermal/voltage) resulting from uncontrolled EV charging on the LV networks (Fig. 1 and Fig. 2) and highlights the importance of a hierarchical control strategy (section III-C). Due to their distinct characteristics, mainly in terms of asset capacity, topology and number of customers, the corresponding EV impacts are discussed separately. To statistically quantify the magnitude of each problem, the average (Avg.) and the standard deviation (Std.) are used. The likelihood of the occurrence of each technical problem is also determined.

\section{A. LV Network 1 (Thermal Issues)}

Fig. 4 highlights that this transformer $(500 \mathrm{kVA})$ may present thermal problems for penetrations larger than $30 \%$. Feeders 4, 5 and 6 may have their capacities exceeded but only for penetrations larger than $70 \%$, i.e., $T x$ problems occur first.

Table III presents the percentage of customers with voltage problems per feeder. Some customers connected to feeders 4, 5 and 6 may experience low voltages for penetrations larger than $70 \%$. On average, however, less than $4 \%$ of the total number of customers may be affected for a $100 \%$ penetration, i.e., voltage problems are not so significant in LV Network 1.

Table IV presents the probabilities of technical problems for LV Network 1. Details are shown for feeders 4, 5 and 6 only, as the others do not present issues at any penetration. The probability of $T x$ thermal problems for a $40 \%$ penetration is not necessarily significant $(13 \%)$. However, they always occur for penetrations higher than $50 \%$.

It can be concluded that the first problem this LV network will face is the thermal overload of the $T x$. Although some feeders may have their capacities exceeded for penetrations larger than $70 \%$, problems on the $T x$ always occur before. This may be related to its initial high utilization factor $(64 \%)$ and the density of customers (see Fig. 1).

\section{B. LV Network 2 (Voltage Issues)}

The utilization factor shown in Fig. 5 highlights that this $T x$ (800 kVA) may present thermal problems only for EV penetrations larger than $70 \%$. However, in terms of feeder utilization, feeders 3, 4 and 5 are constrained at much earlier penetrations (up to 50, 40, and 40\%, respectively). Different from LV Network 1, the capacity of the feeders may be exceeded before this occurs at the $T x$ for this particular LV network.

Table $\mathrm{V}$ shows the percentage of customers with voltage problems. On average, at least one customer in feeder 4 may face significant voltage drops for a penetration as low as $20 \%$. Although not significant, it highlights that for this particular LV network (and similar ones) voltage issues can occur before thermal problems. Indeed, the number of affected customers significantly increases for penetrations larger than $70 \%$. If the standard deviation is considered, up to 30 customers may face significant voltage drops for an $80 \%$ penetration.

Table VI summarizes the probabilities of technical problems for LV Network 2. For a $20 \%$ penetration, the probability of voltage problems in feeders 3 and 4 is not significant ( 0 and 


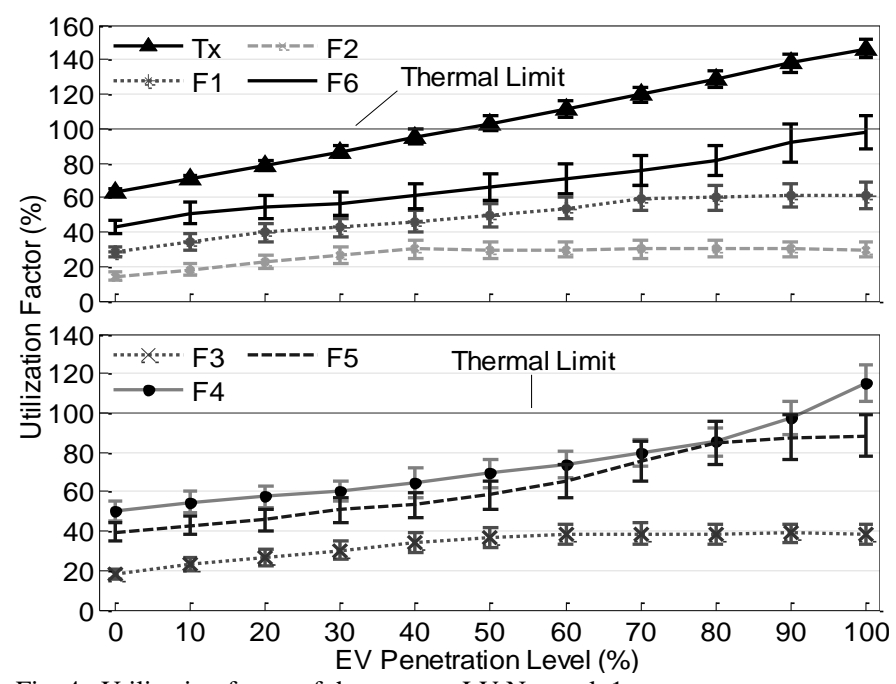

Fig. 4. Utilization factor of the assets - LV Network 1

TABLE III

CUSTOMERS WITH VOLTAGE PROBLEMS PER FEEDER (\%) - LV NETWORK 1

\begin{tabular}{ccccc}
\hline \hline EV Penetration & $70 \%$ & $80 \%$ & $90 \%$ & $100 \%$ \\
\cline { 2 - 5 } Level $(\%)$ & Avg./Std. & Avg./Std. & Avg./Std. & Avg./Std. \\
\hline F4 & $0.0 / 0.0$ & $0.0 / 0.0$ & $0.5 / 2.7$ & $10.7 / 7.2$ \\
F5 & $0.1 / 0.7$ & $1.0 / 2.6$ & $1.5 / 3.5$ & $1.7 / 3.6$ \\
F6 & $0.0 / 0.0$ & $0.0 / 0.0$ & $0.3 / 1.3$ & $1.0 / 1.9$ \\
\hline \hline
\end{tabular}

TABLE IV

PROBABILITY OF TECHNICAL PROBLEMS (\%) - LV NETWORK 1

\begin{tabular}{ccccccccc}
\hline \hline \multirow{2}{*}{ Problem } & \multicolumn{7}{c}{ EV Penetration Level (\%) } \\
\cline { 2 - 9 } & $40 \%$ & $50 \%$ & $60 \%$ & $70 \%$ & $80 \%$ & $90 \%$ & $100 \%$ \\
\hline Tx & 13 & 76 & 100 & 100 & 100 & 100 & 100 \\
\hline Head of & F4 & $0 / 0$ & $0 / 0$ & $0 / 0$ & $1 / 0$ & $3 / 0$ & $41 / 5$ & $95 / 83$ \\
feeder / & F5 & $0 / 0$ & $0 / 0$ & $0 / 0$ & $0 / 3$ & $9 / 19$ & $12 / 20$ & $13 / 21$ \\
Voltage & $\mathrm{F} 6$ & $0 / 0$ & $0 / 0$ & $0 / 0$ & $0 / 0$ & $3 / 0$ & $24 / 6$ & $40 / 25$ \\
\hline \hline
\end{tabular}

$5 \%$, respectively). However, these probabilities significantly increase for an $80 \%$ penetration to 53 and $98 \%$, respectively.

It can be concluded that the first problem this LV network will experience is significant voltage drops mainly for feeder 3 and 4. Different to the previous LV network, another early problem will be feeder thermal issues. This might be related to an initial low utilization of the $T x(48 \%)$ and to the length of the feeders and the density of customers (see Fig. 2).

\section{Implications for the Control Algorithm}

Transformer thermal problems in LV Network 1 occurred first for any penetration level. To mitigate this problem, the control strategy must be designed to consider the aggregated effects of EVs. However, some feeders may face thermal or voltage problems before exceeding the capacity of the $T x$ (e.g., LV Network 2). Understanding this is critical for the design of a hierarchical control strategy where more localized (i.e., per feeder) control actions might be needed for the simultaneous mitigation of thermal and voltage problems.

\section{Proposed CONTROL Algorithm}

This section presents the proposed control algorithm that manages EV charging points to mitigate thermal and voltage problems in LV networks. The control algorithm (based on the Esprit Technology - a patent filed by EA Technology Limited [17]) is currently being trialed in 9 UK LV networks involving 86 EVs [18]. The proposed algorithm, however, has been independently developed by the authors.

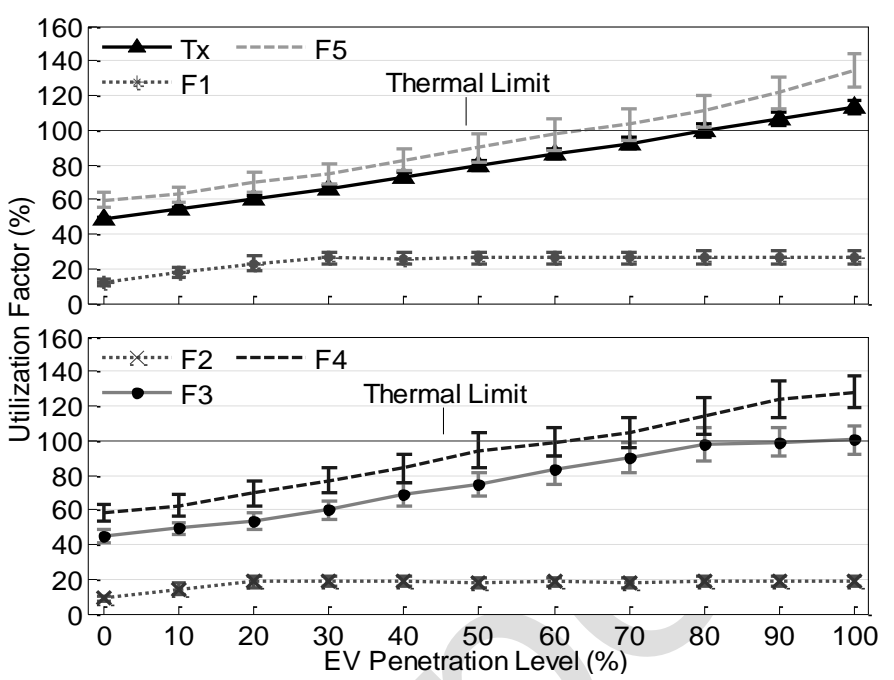

Fig. 5. Utilization factor of the assets - LV Network 2

TABLE V

CUSTOMERS WITH VOLTAGE PROBLEMS PER FEEDER (\%) - LV NETWORK 2

\begin{tabular}{cccccccc}
\hline \hline & \multicolumn{7}{c}{ EV Penetration Level (\%) } \\
\hline & $20 \%-40 \%$ & $50 \%$ & $60 \%$ & $70 \%$ & $80 \%$ & $90 \%$ & $100 \%$ \\
\cline { 2 - 8 } & Avg./Std. Avg./Std. Avg./Std. Avg./Std. Avg./Std. Avg./Std. Avg./Std. \\
\hline F3 & $0.0 / 0.0$ & $0.2 / 0.1$ & $0.4 / 0.5$ & $0.5 / 2.0$ & $4.3 / 5.3$ & $6.2 / 5.6$ & $6.5 / 5.0$ \\
F4 & $0.8 / 0.7$ & $1.2 / 2.9$ & $1.8 / 3.6$ & $2.8 / 4.6$ & $7.6 / 6.8$ & $12.8 / 7.9$ & $16.0 / 6.9$ \\
\hline
\end{tabular}

TABLE VI

Probability of TeChNicAl PRoBlems (\%) - LV Network 2

\begin{tabular}{rcccccccc}
\hline \multirow{2}{*}{ Problem } & \multicolumn{7}{c}{ EV Penetration Level (\%) } \\
\cline { 2 - 8 } & $20 \%-40 \%$ & $50 \%$ & $60 \%$ & $70 \%$ & $80 \%$ & $90 \%$ & $100 \%$ \\
\hline Tx & 0 & 0 & 0 & 2 & 42 & 96 & 100 \\
\hline Head of & $\mathrm{F} 3$ & $0 / 0$ & $0 / 2$ & $3 / 3$ & $14 / 15$ & $40 / 53$ & $45 / 72$ & $48 / 75$ \\
Feeder / & $\mathrm{F} 4$ & $0 / 5$ & $28 / 32$ & $45 / 49$ & $72 / 74$ & $94 / 98$ & $100 / 100$ & $100 / 100$ \\
Voltage & $\mathrm{F} 5$ & $0 / 0$ & $11 / 0$ & $37 / 0$ & $62 / 0$ & $87 / 0$ & $99 / 0$ & $100 / 0$ \\
\hline \hline
\end{tabular}

\section{A. Infrastructure for Implementation}

Fig. 6 shows the implementation architecture of the control strategy. Key infrastructure required (as in the trial) includes: voltage sensors and actuators at the charging points, communication links, voltage and current sensors at the head of the LV feeders (i.e., substation), and a programmable unit (e.g., a PLC) at the substation to host the control algorithm. No direct information such as the SOC from the EVs is required. The simplicity and practicality of this approach is likely to reduce deployment costs and time, making it attractive to DNOs.

\section{B. Control Algorithm}

Fig. 7 shows the flowchart of the control algorithm, which is based on P-controllers [28] all with gain $K$ and considers the hierarchical needs discussed in section III-C. The gains $K$ are defined in section V using the Ziegler-Nichols method [28].

A key feature of the proposed control strategy corresponds to its corrective and preventive approach to disconnect and reconnect EVs, respectively. This provides an adequate balance between the mitigation of technical problems and the comfort of EV users, as further discussed in section V-D.

At every control cycle (i.e., time interval between two consecutive checks), the phase voltages at the busbar, the phase currents at the head of the feeders, the phase charging point voltages, and the status of each EV charging point (i.e., charging) are collected. This information is used to calculate the three-phase $T x$ loading and to update each EV charging time. The latter is done by an internal counter, and it allows defining 


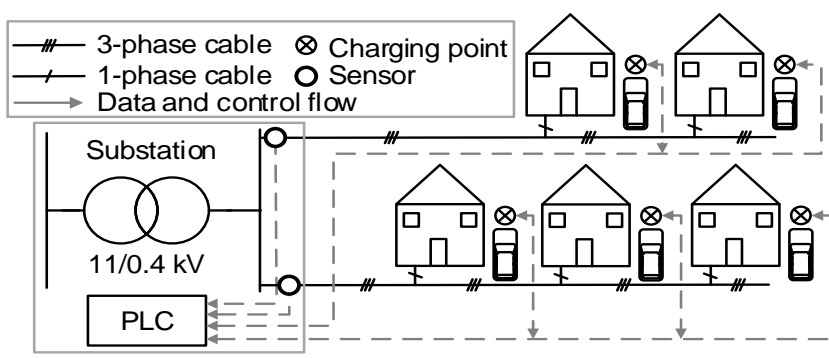

Fig. 6. Practical implementation architecture

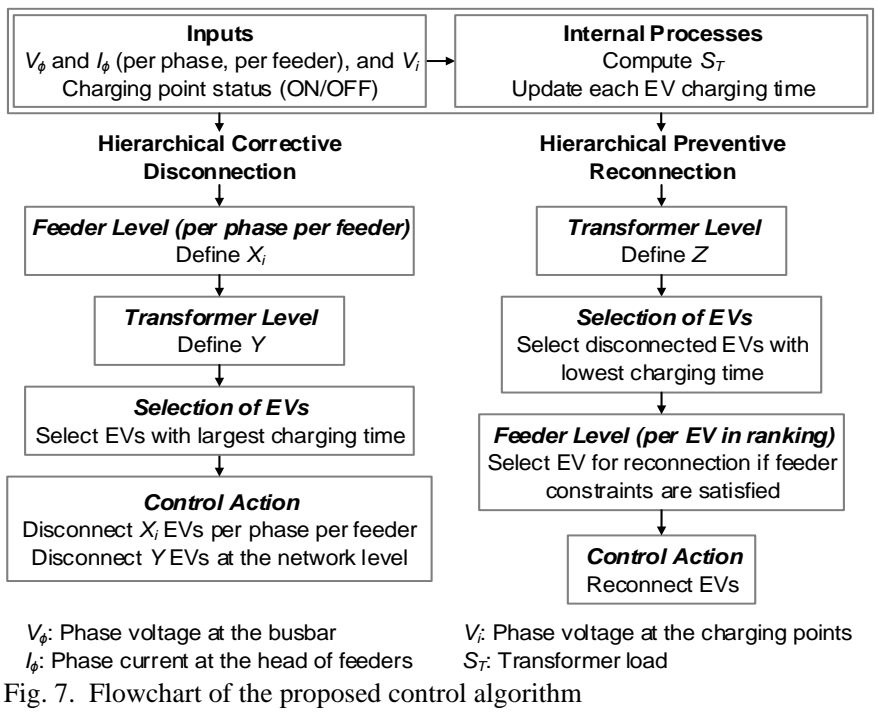

the most suitable EVs to be managed, as below.

1. Hierarchical Corrective Disconnection

If technical problems are detected at the start of the control cycle, the following steps are undertaken to disconnect EVs:

1. Feeder Level. When a phase current , $I_{\phi}$, is above its limit, $I_{\phi}^{m a x}$, a number of EV charging points, $X_{i}$, that must be disconnected in phase $i$ is calculated using a P-controller as follows:

$$
X_{i}=\left\lceil\frac{I_{\phi, i}-I_{\phi, i}^{\max }}{I_{E V}} K\right\rceil
$$

where $[\cdot]$ represents the ceiling function (i.e., roundup), and $I_{E V}$ is the phase current of an EV ( 14A, $230 \mathrm{~V}$ in the case of a Nissan LEAF). In terms of voltage problems, the number of phase charging point voltages (per phase per feeder) below the limit, $V^{\text {min }}$, is determined, and this value is used to define $X_{i}$. Given that this is done per phase, potential issues resulting from unbalances are inherently captured. If both thermal and voltage problems are simultaneously detected in a phase of a feeder at the start of the control cycle, the value of $X_{i}$ is defined as the maximum number of EVs required to solve the thermal or the voltage problem in that particular phase. This process is repeated for each phase of each feeder.

2. Transformer Level. In the hierarchical strategy, the threephase $T x$ loading, $S_{T}$, is then assessed. If this is above the limit, $S_{T}^{\max }$, a number of EVs, $Y$, needed to be disconnected is defined. This is also determined using a P-controller as follows:

$$
Y=\left\{\begin{array}{cc}
0, & \text { if }\left\lceil\frac{S_{T}-S_{T}^{\max }}{S_{E V}} K\right\rceil \leq \sum X_{i} \\
\left\lceil\frac{S_{T}-S_{T}^{\max }}{S_{E V}} K\right\rceil-\sum X_{i}, & \text { if }\left\lceil\frac{S_{T}-S_{T}^{\max }}{S_{E V}} K\right\rceil>\sum X_{i}
\end{array}\right.
$$

where $S_{E V}$ is the EV demand ( $\sim 3.3 \mathrm{~kW}$ for a Nissan LEAF). 3. Selection of EVs. Given that the proposed control strategy does not require the initial SOC, the charging time (calculated by the internal counter) is used as a proxy to determine the cars that are likely to reach full charge. The $X_{i}$ EVs to be disconnected in a given phase and feeder are those with the largest charging times for that particular phase and feeder. When $T x$ thermal problems are detected, the EVs required to disconnect will be chosen based on the largest EV charging times in the entire LV network. Thus, this approach ensures most EV users have a similar charging experience which is not influenced by locational aspects.

4. Control Action. Once the number of EVs needed to mitigate the technical problems is defined, the charging points are disconnected 1 min after. In practice, this delay would correspond to communications and switching actions.

\section{Hierarchical Preventive Reconnection}

If at the start of the control cycle no problems are detected, some previously managed EV charging points are reconnected adopting a preventive approach. The steps below are used.

1. Transformer Level. If the three-phase $T x$ loading is below a security margin, $\alpha$, of its limit, a number of EVs, $Z$, that can be reconnected in the LV network is defined as follows:

$$
Z=\left\lceil\frac{(1-\alpha) S_{T}^{\max }-S_{T}}{S_{E V}} \cdot K\right\rceil
$$

It is important to note that the security margin, $\alpha$, allows catering for the unforeseen arrival of other EVs in the LV network.

2. Selection of EVs. The set of disconnected EVs is ranked according to their charging time (lowest first). This ranking is used sequentially to check whether the reconnection of each $\mathrm{EV}$ is suitable at the feeder level (details below). This process continues until up to $Z \mathrm{EVs}$ are chosen for reconnection.

3. Feeder Level. An EV in the ranking will be chosen for reconnection (i.e., to resume charging) only if the corresponding phase current will be below a margin, $\beta$, of the cable capacity. The maximum number of EVs, $W_{i}^{\max }$, for a given phase, $i$, of a feeder is calculated as follows:

$$
W_{i}^{\max }=\left\lceil\frac{(1-\beta) I_{\phi, i}^{\max }-I_{\phi, i}}{I_{E V}} \cdot K\right\rceil
$$

The margin $\beta$ allows catering for the unforeseen arrival at the feeder level (per phase). Simultaneously, potential phase voltage drops in the feeder are catered for by allowing the reconnection of an EV only if all the phase charging point voltages on the same phase are above $V^{\text {min }}$ plus a security margin $\varepsilon$.

4. Control Action. Once the EVs that can be reconnected are identified, the charging points are switched on 1 min after.

\section{V.APPLICATION OF THE CONTROL ALGORITHM}

This section presents the application of the proposed control algorithm. EV penetrations from 0 to $100 \%$, and four control cycles (i.e., 1, 5, 10 and $30 \mathrm{~min}$ ) are separately examined.

Gain $K$ of the P-controllers: In this section, the gain $K$ of all P-controllers is set as defined by the Ziegler-Nichols method. This heuristic method suggests setting the gain $K$ to $50 \%$ of the optimal gain value $K_{u}$. In the context of the proposed EV control, $K_{u}$ has a unity value to consider all the EVs needed to be disconnected due to a technical issue. Therefore, the gain $K$ 


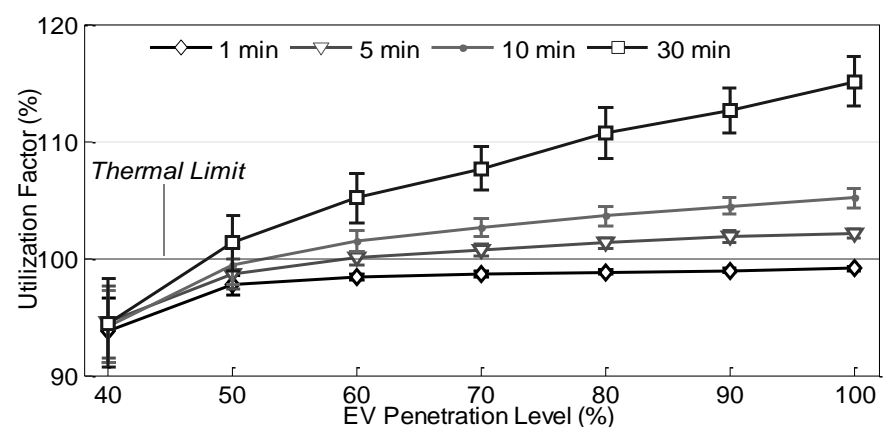

Fig. 8. Transformer utilization factor with control - LV Network 1

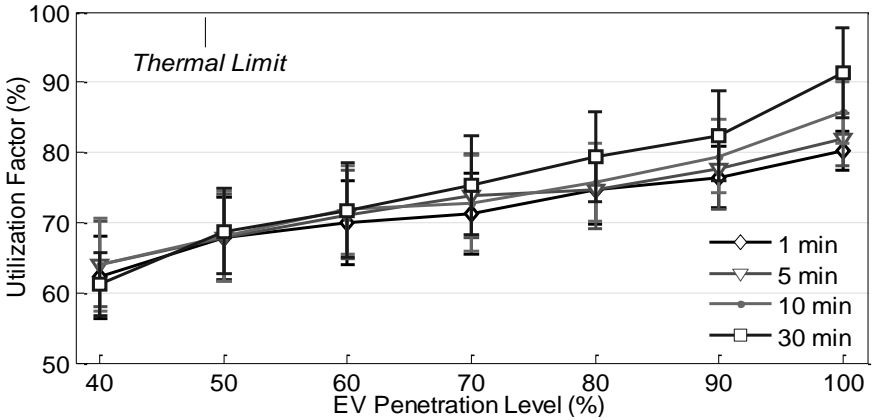

Fig. 9. Utilization factor of Feeder 4 (F4) with control - LV Network 1

TABLE VII

PROBABILITY OF TX OVERLOADS WITH CONTROL (\%) - LV NETWORK 1

\begin{tabular}{cccccccc}
\hline \hline Control & \multicolumn{7}{c}{ EV Penetration Level (\%) } \\
\cline { 2 - 8 } Cycle & $40 \%$ & $50 \%$ & $60 \%$ & $70 \%$ & $80 \%$ & $90 \%$ & $100 \%$ \\
\hline $1 \mathrm{~min}$ & 0 & 0 & 0 & 0 & 0 & 0 & 0 \\
$5 \mathrm{~min}$ & 0 & 0 & 51 & 94 & 100 & 100 & 100 \\
$10 \mathrm{~min}$ & 0 & 46 & 94 & 100 & 100 & 100 & 100 \\
$30 \mathrm{~min}$ & 1 & 73 & 99 & 100 & 100 & 100 & 100 \\
\hline \hline
\end{tabular}

of all P-controllers is set to 0.5 . Theoretically, this decreases the occurrence of under and overshoots [28]. To illustrate the impacts of different gains on the LV network and, crucially, on customers, section V-D presents a sensitivity analysis.

The thermal limits (i.e., $I_{\phi}^{\max }$ and $S_{T}^{\max }$ ) are set to 1 p.u. The voltage limit, $V^{\min }$, is 0.94 p.u., as per UK regulation. To enable the reconnection of EV charging points, the security mar gins $\alpha, \beta$ and $\varepsilon$ are set to 5,10 and $1 \%$, respectively. These settings have been found to be adequate for the studied LV networks in terms of the simultaneous mitigation of technical problems and the comfort of EV users (section V-C). The effects of changing these settings are discussed in section V-D.

\section{A. LV Network 1 (Thermal Issues)}

Fig. 8 shows the $T x$ utilization factor for different EV penetrations and control cycles. Note that $T x$ thermal problems in LV Network 1 occurred first for every EV penetration (section III-A). Table VII presents the probabilities of these overloads. A control cycle of 1 min totally mitigates $T x$ issues even for a $100 \%$ penetration. Although they may occur for control cycles of 5 and $10 \mathrm{~min}$, the $T x$ utilization factor is significantly smaller (106\% at most, Fig. 8), compared to the uncontrolled charging results (see Fig. 4) in which the $T x$ utilization factor reached $150 \%$ for a full adoption $(100 \%)$.

The utilization factor of the most loaded feeder (Feeder 4, F4) shown in Fig. 9 highlights that thermal problems in this feeder (and indeed all the others) are mitigated, even for a $100 \%$ penetration and a 30-min control cycle. The mitigation of feeder thermal issues in LV Network 1 is the result of managing EV charging points at the transformer level, where the

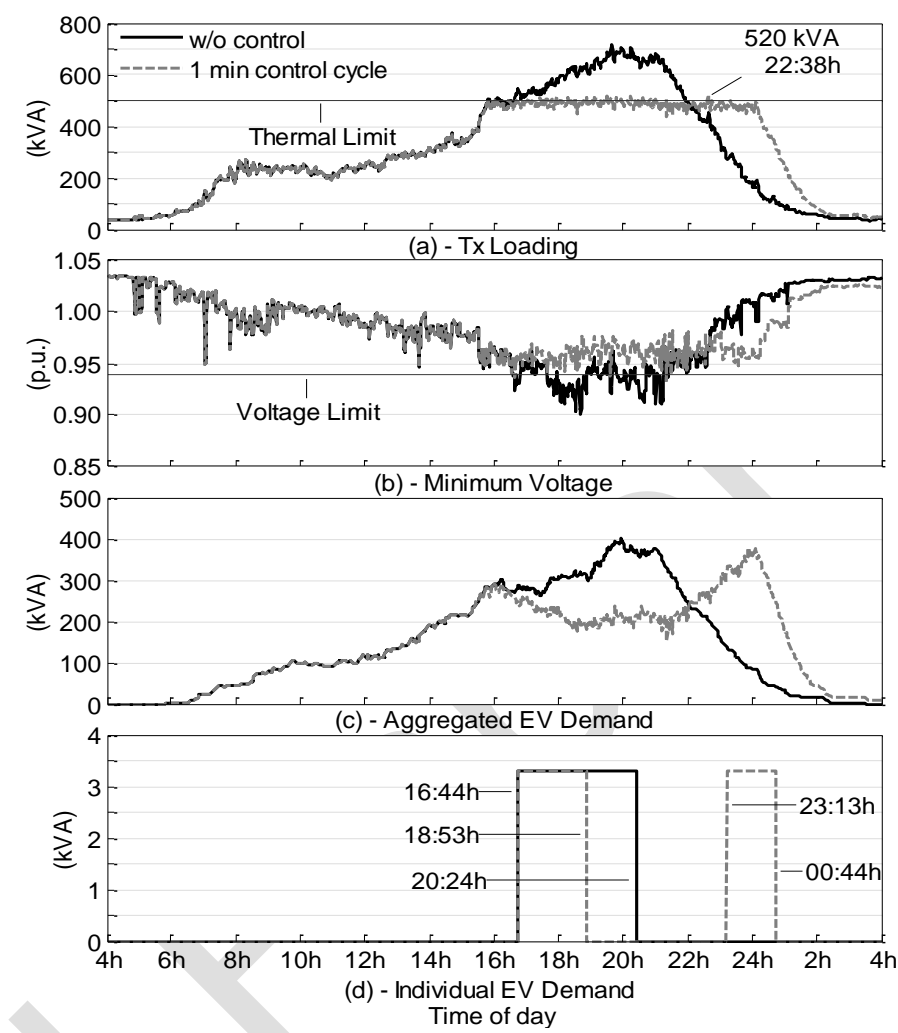

Fig. 10. 24-hour operation and performance example - LV Network 1

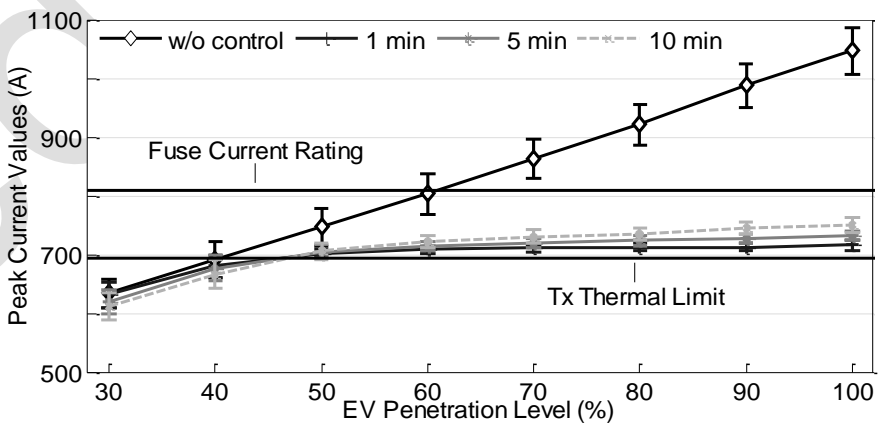

Fig. 11. Peak values for different time intervals - Tx LV Network 1

first problem occurs. This has also avoided voltage issues, even for a $100 \%$ penetration and a control cycle of $30 \mathrm{~min}$.

Whilst $T x$ overloads in LV Network 1 are totally mitigated for 1-min control cycles, it can be concluded that control cycles of 5 and $10 \mathrm{~min}$ can be equally effective. This is important because it highlights that latency and IT needs related to the monitoring data can be relaxed. In addition, longer control cycles lead to less control actions and, therefore, this can have a positive effect on EV users (see section V-C).

1. 24-Hour Operation and Performance Example

To illustrate the operation of EV charging points and network performance during a day, a comparison is carried out between the cases without and with control (1-min) considering a $100 \%$ EV penetration (arbitrarily selected from the Monte Carlo analysis). The $T x$ loading shown in Fig. 10(a) demonstrates that the proposed control effectively reduces the utilization factor (from 136 to 99\%). As shown in Fig. 10(b), which presents the minimum phase voltage every minute, this also improves voltages across the network. To achieve this, the control algorithm managed a total of $190 \mathrm{EV}$ charging points (54.1\%) mostly between 16 and 24h (Fig. 10(a)). The effect of this charging point management on the aggregated EV de- 
mand can be seen in Fig. 10(c) where the peak without control is shifted to later hours. This effect is also shown in Fig. 10(d) where the charging of a single EV is presented. It can be seen that the disconnection happens around the time of high aggregated EV demand (i.e., most EV users are charging at home). Crucially, when the overall network demand is much lower, this EV is reconnected, resuming the charging process until the expected charging time is reached (3hours $40 \mathrm{~min}$ ).

\section{Peak Values and Time Intervals}

Peak current values higher than the capacity of the assets may occur before or after an action from the control algorithm. This is because of the corresponding average values (according to the control cycle) or the unforeseen arrivals of EVs.

For instance, although the $T x$ utilization factor shown in Fig. 10(a) for a 1-min control cycle is $99 \%$, it is possible to observe a peak value of $520 \mathrm{kVA}$ (i.e., $104 \%$ ) at $22: 38 \mathrm{~h}$. Although this peak (709 A) and all others (60 in total) are higher than the $T x$ capacity (500 kVA, $693 \mathrm{~A}$ ), it should be noted that their magnitudes are not significant given that they are in fact distant from the current rating of the fuse used in the actual substation ( 810 A, fuse type 560SJ31 [29]).

From a probabilistic perspective, Fig. 11 shows the peaks on the $T x$ considering different time intervals which are equal to the corresponding control cycle (e.g., peak values for a 5min control cycle use 5 min average data). Clearly, even for a $100 \%$ penetration and a control cycle of up to $10 \mathrm{~min}$, the peak current (average) values are below the fuse current rating.

\section{B. LV Network 2 (Voltage Issues)}

In this case, the first technical problem to occur is due to voltage drops in feeders 3 and 4. Table VIII shows that all customer voltages are above the limit when 1 and 5-min control cycles are used. For 10-min control cycles, voltage issues may occur in feeders 3 and 4 - with probability no higher than $9 \%$, but only for penetrations larger than 70 and $60 \%$, respectively (see Table IX). On average, however, less than one customer in this LV network may present voltage issues when every house has an EV. As for 30-min control cycles, Table VIII highlights that voltage issues may occur for penetrations higher than $50 \%$. More importantly, the scale of affected customers could increase up to $2.5 \%$ for a full EV adoption.

In terms of thermal problems, the utilization factor shown in Fig. 12 highlights that the transformer will not present issues with control cycles of up to $10 \mathrm{~min}$. As for the head of the feeders, Fig. 13 shows that thermal problems are fully mitigated for 1-min control cycles. Although for 5 and 10-min control cycles the capacity of the feeders may be exceeded for high penetrations (probabilities included in Table IX), the magnitude is small. For instance, for a $100 \% \mathrm{EV}$ penetration, the utilization factor of feeder 5 (149 customers) is on average $104 \%$ for 10-min (Fig. 13) compared to the uncontrolled charging results (Fig. 5) in which this value reached $138 \%$.

Voltage and thermal problems in LV Network 2 have been mitigated using control cycles of up to $10 \mathrm{~min}$. Therefore, it can be concluded that the proposed control strategy is successful in simultaneously mitigating both thermal and voltage problems on the two studied LV networks.

\section{Effects on Customers}

Assessing the impacts that a control strategy has on cus-

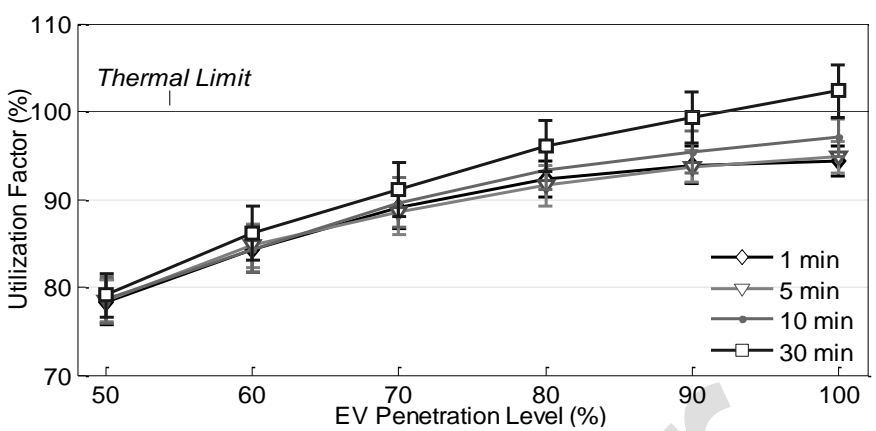

Fig. 12. Transformer utilization factor with control - LV Network 2

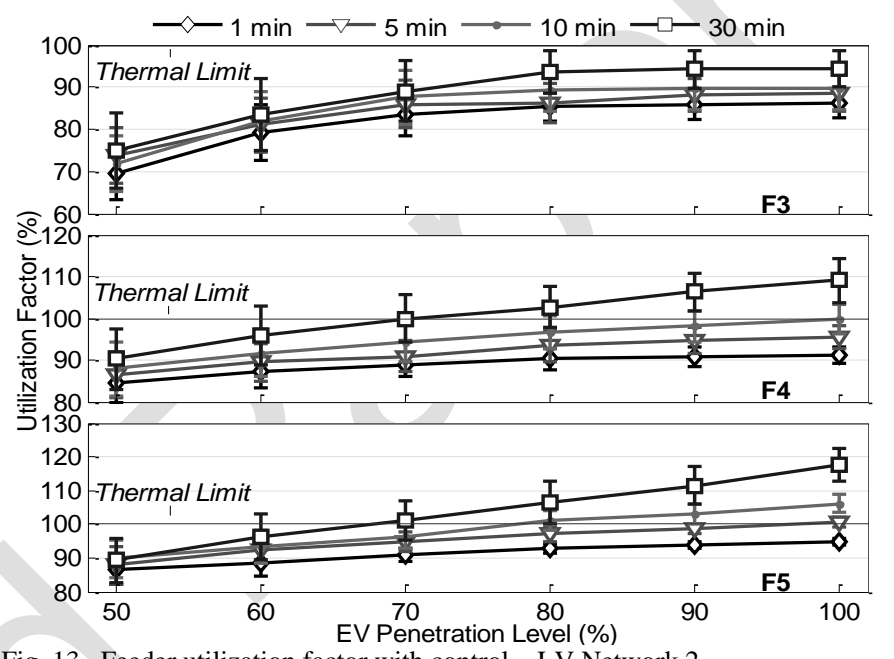

Fig. 13. Feeder utilization factor with control - LV Network 2

TABLE VIII

CUSTOMERS WITH VOLTAGE PROBLEMS WITH CONTROL (\%) - LV NETWORK 2

\begin{tabular}{lcccccc}
\hline \hline \multirow{2}{*}{$\begin{array}{c}\text { Control } \\
\text { Cycle }\end{array}$} & $50 \%$ & $60 \%$ & $70 \%$ & $80 \%$ & $90 \%$ & $100 \%$ \\
\cline { 2 - 7 } & Avg./Std. & Avg./Std. & Avg./Std. & Avg./Std. & Avg./Std. & Avg./Std. \\
\hline 1,5 min & $0 / 0$ & $0 / 0$ & $0 / 0$ & $0 / 0$ & $0 / 0$ & $0 / 0$ \\
\hline $10-\mathrm{min}$ & $0 / 0$ & $0 / 0$ & $0.02 / 0.12$ & $0.03 / 0.14$ & $0.07 / 0.26$ & $0.22 / 0.38$ \\
$30-\mathrm{min}$ & $0 / 0$ & $0.03 / 0.17$ & $0.08 / 0.29$ & $0.30 / 0.50$ & $0.70 / 0.78$ & $1.43 / 0.93$ \\
\hline
\end{tabular}

TABLE IX

PRobability of FEeder PRoBlems With CONTROL (\%) - LV NeTWORK 2

\begin{tabular}{cllccccc}
\hline \hline \multirow{2}{*}{$\begin{array}{c}\text { Control } \\
\text { Cycle }\end{array}$} & Problem & \multirow{2}{*}{ Feeder } & \multicolumn{5}{c}{ EV Penetration Level (\%) } \\
\cline { 3 - 8 } 5 & & $60 \%$ & $70 \%$ & $80 \%$ & $90 \%$ & $100 \%$ \\
\hline \multirow{3}{*}{ min } & Head of & F3 & $0 / 0$ & $0 / 0$ & $0 / 0$ & $0 / 0$ & $0 / 0$ \\
& Feeder / & F4 & $0 / 0$ & $0 / 0$ & $1 / 0$ & $2 / 0$ & $2 / 0$ \\
& Voltage & F5 & $0 / 0$ & $0 / 0$ & $3 / 0$ & $9 / 0$ & $35 / 0$ \\
\hline \multirow{3}{*}{10 min } & Head of & F3 & $0 / 0$ & $0 / 0$ & $0 / 2$ & $0 / 4$ & $0 / 7$ \\
& Feeder / & F4 & $1 / 0$ & $9 / 2$ & $19 / 1$ & $30 / 3$ & $52 / 9$ \\
& Voltage & F5 & $1 / 0$ & $13 / 0$ & $49 / 0$ & $65 / 0$ & $86 / 0$ \\
\hline \multirow{3}{*}{30 min } & Head of & F3 & $2 / 1$ & $5 / 1$ & $13 / 10$ & $14 / 19$ & $15 / 20$ \\
& Feeder / & F4 & $30 / 2$ & $48 / 7$ & $69 / 18$ & $93 / 41$ & $94 / 80$ \\
& Voltage & F5 & $29 / 0$ & $59 / 0$ & $86 / 0$ & $97 / 0$ & $100 / 0$ \\
\hline \hline
\end{tabular}

tomers is critical to determine if the expectations of comfort are met [16]. Understanding this is crucial not only to promote customer acceptance but also to adopt the most adequate control settings. Quantifying the number of switching actions (defined as the total variation, i.e., TV, [28]) is also important as a proxy to assess the potential effects on the EV battery.

In the uncontrolled charging, EVs are charged as required, i.e., they are not disconnected and their $\mathrm{TV}=0$. Hence, the charging time to reach a given charge is equal to the expected (by users) charging time. However, this may not happen if a controlled charging strategy is used, as some EVs must be managed to avoid technical problems (i.e., thermal/voltage).

In an effort to estimate the impact that the control strategy 
TABLE $X$

COMFORT METRIC TO ASSESS THE EFFECTS OF CUSTOMERS

\begin{tabular}{ccccccccccc}
\hline \hline CIL & $\mathbf{0}$ & $\mathbf{1}$ & $\mathbf{2}$ & $\mathbf{3}$ & $\mathbf{4}$ & $\mathbf{5}$ & $\mathbf{6}$ & $\mathbf{7}$ & $\mathbf{8}$ & $\mathbf{9}$ \\
\cline { 2 - 11 } Additional & & $1-$ & $26-$ & $51-$ & $76-$ & $101-$ & $126-$ & $151-$ & $176-$ & \multirow{2}{*}{200} \\
Charging Time (\%) & 0 & 25 & 50 & 75 & 100 & 125 & 150 & 175 & 200 & \\
\hline \hline
\end{tabular}

TABLE XI

UNAFFECTED CUSTOMERS, CIL $=0(\%)-$ LV NETWORK 1

\begin{tabular}{cccccccc}
\hline \hline Control & \multicolumn{7}{c}{ EV Penetration Level (\%) } \\
\cline { 2 - 7 } Cycle & $40 \%$ & $50 \%$ & $60 \%$ & $70 \%$ & $80 \%$ & $90 \%$ & $100 \%$ \\
\hline $1 \mathrm{~min}$ & 99 & 87 & 72 & 59 & 50 & 44 & 40 \\
$5 \mathrm{~min}$ & 99 & 89 & 77 & 67 & 60 & 56 & 51 \\
$10 \mathrm{~min}$ & 100 & 91 & 80 & 71 & 63 & 59 & 54 \\
$30 \mathrm{~min}$ & 100 & 95 & 85 & 76 & 70 & 64 & 59 \\
\hline \hline
\end{tabular}

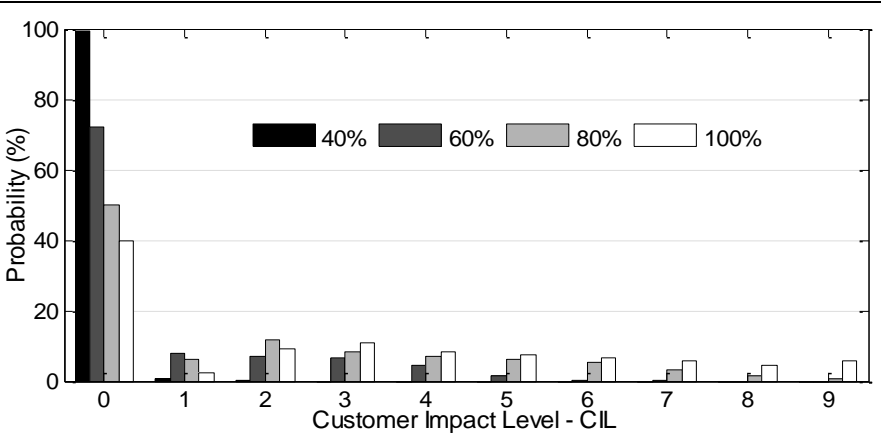

Fig. 14. Probability of CILs for 1-min control cycle - LV Network 1

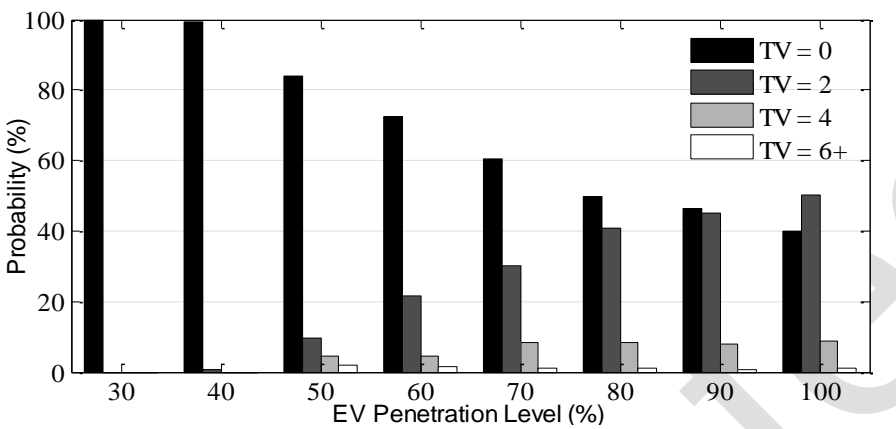

Fig. 15. Probability of various TVs for 1-min control cycle - LV Network 1

has on customers, this work introduces a metric: the customer impact level (CIL). The CIL indicates the ratio between the charging time taken by the EV to reach the required level of charge during the controlled charging and the time expected by the user to reach the same level of charge, i.e., the time that it would have taken in the uncontrolled charging.

Table X shows 10 CILs (i.e., 0-9) that are used to examine the charging time delays. For instance, an EV that is expected to receive $1 \mathrm{~h}$ charge will have a CIL 2 if it (after management) required $90 \mathrm{~min}$ (50\% more) to reach the same charge.

\section{LV Network 1}

Table XI shows the percentage of unaffected customers (i.e., $\mathrm{CIL}=0$ ) for different $\mathrm{EV}$ penetrations and control cycles. For 1-min control cycle, Fig. 14 details the probability that a customer in LV Network 1 has of having each CIL for various EV penetrations. As it is expected, the higher the penetration, the lower the number of unaffected customers. In addition, the longer the control cycle, the higher the number of unaffected customers, i.e., long control cycles disconnect less EVs.

It should be noted that these values represent the probability of reaching the expected charging level as if there was no management, and thus, they could be used to promote the deployment of the control strategy as well as the EV adoption.

In particular, it is important to highlight that 10-min control cycles lead, on average, to a $10 \%$ increase in the number of
TABLE XII

UNAFFECTED CUSTOMERS, CIL $=0(\%)-$ LV NETWORK 2

\begin{tabular}{cccccccccc}
\hline \hline Control & \multicolumn{8}{c}{ EV Penetration Level (\%) } \\
\cline { 2 - 10 } Cycle & $20 \%$ & $30 \%$ & $40 \%$ & $50 \%$ & $60 \%$ & $70 \%$ & $80 \%$ & $90 \%$ & $100 \%$ \\
\hline 1 min & 99 & 98 & 97 & 94 & 91 & 85 & 77 & 68 & 62 \\
5 min & 99 & 99 & 98 & 95 & 92 & 87 & 80 & 73 & 68 \\
$10 \mathrm{~min}$ & 100 & 100 & 98 & 96 & 94 & 89 & 83 & 77 & 71 \\
$30 \mathrm{~min}$ & 100 & 100 & 99 & 98 & 97 & 94 & 89 & 84 & 79 \\
\hline \hline
\end{tabular}

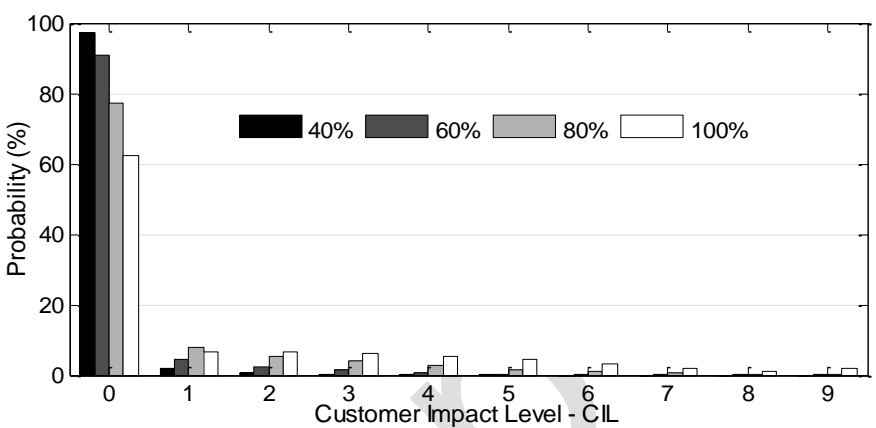

Fig. 16. Probability of CILs for 1-min control cycle - LV Network 2

unaffected customers when compared to 1-min case. Although 30-min control cycles are more beneficial for the EV users, significant technical problems might still occur (section V-A).

It should also be noted that the values in Table XI represent the percentage of $\mathrm{EVs}$ with a $\mathrm{TV}=0$, i.e., $\mathrm{EV}$ charging points that are never disconnected/reconnected. This quantification is important because it highlights the extent to which the control strategy might affect the life span of EV batteries.

Fig. 15 shows the probability of various TV values for a 1min control cycle and different EV penetrations. The TV is zero or two in more than $88 \%$ of the cases. Only for EV penetrations higher than $80 \%$, more than half of the EVs are managed (i.e., disconnected/reconnected at some point).

\section{LV Network 2}

For LV Network 2, Table XII shows the percentage of unaffected customers for different penetration levels and control cycles. For a control cycle of $1 \mathrm{~min}$ and different penetrations, Fig. 16 examines the probability of each CIL. Similar to LV Network 1, the longer the control cycle, the higher the number of unaffected customers $(\mathrm{CIL}=0)$. Moreover, the higher the penetration level, the higher the number of affected customers.

It is interesting to note here that the percentage of unaffected customers increases in LV Network 2. This is due to the localized impacts (i.e., per feeder) that require a targeted action. In practice, this means that EVs in feeders without technical problems will not be disconnected. Finally, the probability of the TV in LV Network 2 has a similar behavior as before, i.e., most of the EV charging points are not managed (i.e., $\mathrm{TV}=0)$ or were disconnected only once $(\mathrm{TV}=2)$.

\section{D.Effects of Different Control Settings}

The proposed control algorithm totally mitigates technical problems in the two studied LV networks when a control cycle of $1 \mathrm{~min}$ is adopted. However, this requires extensive use of the communication infrastructure, data management, and it results in longer EV charging times. A compromise can be achieved by adopting longer control cycles, e.g., 5 and $10 \mathrm{~min}$. Although small overloads might occur, the percentage of unaffected customers (i.e., $\mathrm{CIL}=0$ ), will significantly increase. For example, Fig. 8 shows for a $100 \%$ EV penetration and control cycles of 5 and $10 \mathrm{~min}$ that the average $T x$ utilization 
TABLE XIII

HIERARCHICAL PREVENTIVE DISCONNECTION RESULTS - LV NETWORK 1

\begin{tabular}{|c|c|c|c|c|c|c|c|}
\hline \multirow{2}{*}{$\begin{array}{c}\text { Control } \\
\text { Cycle }\end{array}$} & \multirow{2}{*}{ Feature } & \multicolumn{6}{|c|}{ EV Penetration Level (\%) } \\
\hline & & 50 & 60 & 70 & 80 & 90 & 100 \\
\hline \multirow{3}{*}{$5 \mathrm{~min}$} & $T x$ UF (\%) & 96.3 & 97.4 & 97.9 & 98.4 & 98.8 & 99.2 \\
\hline & Prob. (\%) & 0 & 0 & 0 & 0 & 0 & 3 \\
\hline & $\mathrm{CIL}=0(\%)$ & 83 & 69 & 62 & 55 & 52 & 47 \\
\hline \multirow{3}{*}{$10 \mathrm{~min}$} & $T x$ UF (\%) & 94.8 & 96.2 & 97.0 & 97.8 & 98.5 & 99.1 \\
\hline & Prob. $(\%)$ & 0 & 0 & 0 & 0 & 0 & 12 \\
\hline & $\mathrm{CIL}=0(\%)$ & 80 & 69 & 62 & 56 & 52 & 48 \\
\hline
\end{tabular}

TABLE XIV

EFFECTS OF DIFFERENT GAIN VALUES - LV NETWORK 2

\begin{tabular}{|c|c|c|c|c|c|c|c|c|c|c|}
\hline \multirow{2}{*}{$\begin{array}{c}\text { Control } \\
\text { Cycle }\end{array}$} & \multirow{2}{*}{ Gain $K$} & \multirow{2}{*}{ Feature } & \multicolumn{8}{|c|}{ EV Penetration Level (\%) } \\
\hline & & & 30 & 40 & 50 & 60 & 70 & 80 & 90 & 100 \\
\hline \multirow{4}{*}{$5 \mathrm{~min}$} & \multirow{2}{*}{0.25} & No. of Feeders & 0 & 0 & 1 & 2 & 2 & 3 & 3 & 3 \\
\hline & & $\mathrm{CIL}=0(\%)$ & 99 & 99 & 98 & 95 & 90 & 83 & 76 & 72 \\
\hline & \multirow{2}{*}{0.75} & No. of Feeders & 0 & 0 & 0 & 0 & 0 & 0 & 1 & 1 \\
\hline & & $\mathrm{CIL}=0(\%)$ & 99 & 98 & 95 & 91 & 86 & 79 & 72 & 66 \\
\hline \multirow{4}{*}{$10 \mathrm{~min}$} & \multirow{2}{*}{0.25} & No. of Feeders & 0 & 0 & 2 & 2 & 3 & 3 & 3 & 3 \\
\hline & & $\mathrm{CIL}=0(\%)$ & 100 & 100 & 98 & 95 & 90 & 86 & 79 & 74 \\
\hline & \multirow{2}{*}{0.75} & No. of Feeders & 0 & 0 & 0 & 0 & 0 & 1 & 1 & 1 \\
\hline & & $\mathrm{CIL}=0(\%)$ & 99 & 98 & 96 & 93 & 89 & 83 & 75 & 70 \\
\hline
\end{tabular}

factor of LV Network 1 is 102 and $105 \%$, respectively. On the other hand, Table XI shows that the number of unaffected EV users in the same LV network increases by 11 and 14\%, compared to a 1-min control cycle, respectively.

Thermal and voltage problems can also be mitigated for control cycles longer than 1 min by undertaking a hierarchical preventive disconnection approach. As an example for control cycles of 5 and $10 \mathrm{~min}$ in LV Network 1, Table XIII shows the results of disconnecting EVs when the $T x$ utilization factor (Tx UF) reaches 97 and 95\%, respectively. These new settings (effectively using a security margin) significantly reduce the average $T x$ UF and the probability of $T x$ overloads. However, this hierarchical preventive disconnection approach increases the number of affected customers in more than $4 \%$, and even $11 \%$ in some cases.

A gain $K=0.5$ proved to be for the studied LV networks an adequate compromise between the mitigation of technical problems and the customer comfort, as detailed above. Increasing this gain could also minimize technical issues, but, again, it results in more affected customers. Table XIV shows the results of setting $K$ to 0.25 and 0.75 in LV Network 2. It can be observed that a higher gain $K$ reduces the number of feeders with probability of overloads higher than zero. However, this reduces in $2 \%$ the number of unaffected customers.

\section{OPtimizATION-BASED EV MANAGEMENT}

To understand the extent to which the proposed control algorithm provides effective network management and adequate comfort levels for EV users, this section provides a comparison with an ideal, optimization framework driven by an AC Optimal Power Flow (OPF). For this purpose, and given the unbalance nature of LV networks, the approach proposed in [30] is extended to a three-phase AC OPF.

\section{A. Problem Formulation}

The disconnection and reconnection of EV charging points is determined in this case by the AC OPF. Different from the proposed control algorithm, this optimization engine requires the full LV network model (i.e., topology, customer phase connection, and line impedances). In addition, significant ob- servability is needed to mimic the state of the network, for instance, using smart meter data per customer.

To determine the optimal status (on/off), $\psi_{e v}$, of the EV charging points (set $E V$ indexed by $e v$ ), the objective function of the $\mathrm{AC} \mathrm{OPF}$ is formulated to minimize the number of $\mathrm{EV}$ disconnections as given in (5).

$$
\min \sum_{e v \in E V}\left(1-\psi_{e v}\right) \rho^{\left(1-S O C_{e v}\right)}
$$

where $\rho$ is a constant larger than one. To define the EVs to be managed, the SOC is used. An EV with high SOC is disconnected first, and one with high SOC is reconnected last. This strategy intends to minimize the impact on EV users. This objective is subject to the typical AC OPF constraints (i.e., Kirchhoff's voltage and current laws) as well as voltage and thermal limits. For simplicity, the implemented AC OPF neglects the mutual effects between phases.

For the $T x$ and each line (all represented by the set $L$ indexed by $l$ ), the active $f_{l, \phi}^{(1,2), P}$ and reactive power $f_{l, \phi}^{(1,2), Q}$ injections in each phase (set $\Phi$ indexed by $\phi$ ) at both ends of $l$ (denoted by 1 and 2) are calculated according to the Kirchhoff's voltage expressions for active, $f_{l, \phi, K V L}^{(1,2),(P)}(V, \delta)$, and reactive power, $f_{l, \phi, K V L}^{(1,2),(Q)}(V, \delta)$, respectively. This is shown below:

$$
f_{l, \phi}^{(1,2),(P, Q)}=f_{l, \phi, K V L}^{(1,2),(P, Q)}(V, \delta) \quad \forall l \in L, \forall \phi \in \Phi
$$

where $V$ and $\delta$ are the voltage magnitude and angle. The balance of active and reactive power at each bus (set $B$ indexed $b$ ), and for each phase $\phi$ are formulated as follows:

$$
\begin{aligned}
& \sum_{e v \in E V \mid \gamma_{e v}=b, \Phi_{e v}=\phi} \psi_{e v} S_{e v}+\sum_{d \in D \mid \gamma_{d}=b, \Phi_{d}=\phi} p_{d}+\sum_{l \in L \mid \gamma_{l}^{(1,2)}=b} f_{l, \phi}^{(1,2), P}=\sum_{x \in X \mid \gamma_{x}=b} p_{x, \phi} \\
& \sum_{d \in D \mid \gamma_{d}=b, \Phi_{d}=\phi} q_{d}+\sum_{l \in L \mid \gamma_{l}^{(1,2)}=b} f_{l, \phi}^{(1,2), Q}=\sum_{x \in X \mid \gamma_{x}=b} q_{x, \phi}
\end{aligned}
$$

where $\left(p_{x, \phi}, q_{x, \phi}\right)$ are the active and reactive power imported/exported from the grid (set $X$ indexed by $x$ ) and whose values are within limits $\left(p_{x}^{(-,+)}, q_{x}^{(-,+)}\right) ;\left(p_{d}, q_{d}\right)$ are the active and reactive power demand of customers (set $D$ indexed by $d$ ), $\gamma_{u}$ maps the location of each element $(u \subset\{e v, d, l, x\})$ to its corresponding bus, $b$, and $\phi_{v}$ maps the phase of loads and EVs $(v \subset\{d, e v\})$ to its associated phase $\phi$.

The OPF is triggered if the thermal limits (head of the feeder or $T x$ ) are exceeded, $f_{l, \phi}^{+}$or if any phase customer voltage $\left(V_{b, \phi}\right)$ is below the statutory limit, $V^{\text {min }}$. To reconnect EVs, similarly to the proposed control algorithm, security margins $(\alpha, \beta, \varepsilon)$ are adopted for thermal and voltage constraints:

$$
\begin{gathered}
\left(f_{l, \phi}^{(1,2), P}\right)^{2}+\left(f_{l, \phi}^{(1,2), Q}\right)^{2} \leq\left(\lambda_{l} f_{l, \phi}^{+}\right)^{2} \quad \forall l \in L, \forall \phi \in \Phi \\
\left(V^{\min }+\varepsilon\right) \leq V_{b, \phi} \quad \forall b \in B, \forall \phi \in \Phi
\end{gathered}
$$

where $\lambda_{l}$ is the corresponding security margin $(\alpha$ or $\beta)$.

To solve the above complex Mixed Integer Non-Linear Programming problem (MINLP), the binary decision variables associated with the status of each EV charging point, $\psi_{e v}$, are relaxed and considered as continuous variables bounded between zero and one. Therefore, the status of each EV charging 


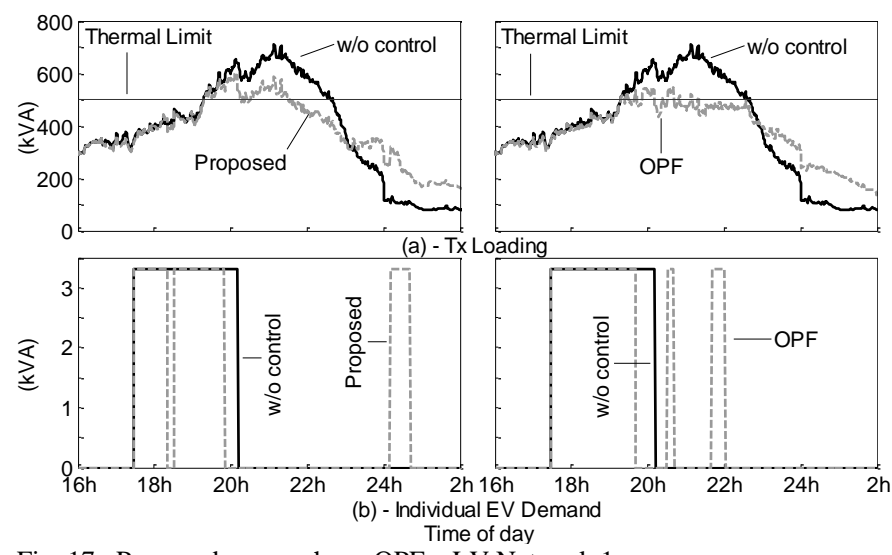

Fig. 17. Proposed approach vs. OPF - LV Network 1

TABLE XV

COMPARISON PROPOSED APPROACH VS. OPF - LV NETWORK 1

\begin{tabular}{cccccccc}
\hline \hline \multirow{2}{*}{ Feature } & Control & \multicolumn{6}{c}{ EV Penetration Level $(\%)$} \\
\cline { 3 - 7 } & Approach & 50 & 60 & 70 & 80 & 90 & 100 \\
\hline \multirow{2}{*}{ Tx UF (\%) } & w/o control & 88 & 95 & 103 & 111 & 118 & 132 \\
& Proposed & 88 & 94 & 98 & 102 & 104 & 107 \\
& OPF & 87 & 92 & 95 & 98 & 99 & 102 \\
\hline \multirow{2}{*}{$\mathrm{CIL}=0(\%)$} & Proposed & 100 & 98 & 94 & 88 & 81 & 69 \\
& OPF & 93 & 89 & 87 & 80 & 73 & 67 \\
\hline \hline
\end{tabular}

point requires a post-optimization adjustment. To do so, EV charging points whose status variable is smaller than a threshold, $\mu$, are disconnected. To avoid excessive reconnections or unnecessary disconnections due to this approximation, the optimization is done iteratively so $\mu$ is progressively increased (from 0.1 to 1.0 in steps of 0.1 ) until the status of all EV charging points are truly binary. For a given iteration, those with $\psi_{e v}<\mu$ are enforced to be disconnected $\left(\psi_{e v}=0\right)$ in the next one. This allows the OPF to ultimately find unity values of $\psi_{e v}$ to charging points that do not lead to technical issues.

\section{B. Comparison}

This section compares the performance of the OPF-based optimization engine and the proposed control algorithm considering LV Network 1 ( $T x$ problems occur first). For clarity, a single deterministic case (for different $\mathrm{EV}$ penetrations) is studied adopting only one control cycle (10-min).

The modeling language AIMMS [31] is used to formulate and solve the AC OPF and solved using the CONOPT 3.14V NLP solver. OpenDSS provides the network state at each control cycle before and after applying the optimal set points [20].

Table XV summarizes the $T x$ utilization factor ( $T x$ UF) and the percentage of unaffected $\mathrm{EV}$ users (i.e., $\mathrm{CIL}=0$ ) for different EV penetrations. In much more detail, for a $100 \% \mathrm{EV}$ penetration, Fig. 17 compares the 1-min Tx loading and the individual demand of a charging point. As expected, the OPF results in a better management of the $T x$ loading (lower $T x$ $U F)$. Nonetheless, this improvement is at most 5\%; which highlights that the proposed approach can achieve a very effective management without the need of extensive network and monitoring data. From the customer perspective, Table XIII shows that the proposed approach resulted, on average, in $6 \%$ less disconnections (i.e., more EV users with $\mathrm{CIL}=0$ ).

\section{DISCUSSION}

The control approach checks voltage issues per phase per feeder and thus captures voltage drop problems that may also result from unbalances. This includes unbalance issues that might occur due to $\mathrm{EV}$ disconnections or reconnections.

The P-controller proved to be an effective option in managing EV charging points. More advanced controllers, such as PI, could be implemented by considering past errors. This, however, might require shorter control cycles and, thus, could affect more EV customers (e.g., higher TV or CIL).

To cater for different EV demands due to different battery sizes and charging modes, the proposed approach can be adapted by considering in the disconnection/reconnection ranking both the time and the corresponding EV demand.

Finally, although the proposed control algorithm focuses on LV networks, integrated MV-LV studies can be undertaken to understand the wider EV impacts and corresponding solutions to adequately manage regional-scale EV penetrations (e.g., [13]). In addition, the coordinated use of other control devices (e.g., on-load tap changers, capacitor banks) can be explored.

\section{VIII.CONCLUSIONS}

This work has proposed an implementable, centralized control algorithm, currently being trialed in $9 \mathrm{UK}$ residential $\mathrm{LV}$ networks involving $86 \mathrm{EVs}$, that uses limited information to manage EV charging points to mitigate simultaneous thermal and voltage problems in LV networks. Two real three-phase with single-phase customer connection points UK LV networks were used to demonstrate the effectiveness of the control algorithm (investigated for four control cycles). A Monte Carlo approach (adopting 1-min resolution data) was used to cater for the uncertainties related to household demand as well as EV demand and location. Crucially, the charging time delays and the number of switching actions were investigated to understand the effects on the comfort of EV users.

The control strategy successfully mitigated technical problems (i.e., thermal/voltage) on the examined LV networks, particularly with control cycles of up to $10 \mathrm{~min}$ (even for a $100 \%$ penetration). It was shown, however, that the use of 5 and 10-min control cycles significantly improves the comfort of EV users (in 11 and 14\% for a full adoption) and reduces the number of switching actions, compared to a 1-min control cycle. Although using these control cycles may result in small asset overloads, they have the potential to promote customer acceptance and, hence, help accelerating the adoption of EVs. Finally, a comparison with an ideal optimization framework based on a three-phase AC OPF highlighted that the proposed algorithm is as effective whilst using limited information. Thus, the practicality and effectiveness makes the proposed approach more attractive to DNOs as a solution towards the transition to complex Smart Grid schemes.

Given its centralized approach, the control strategy relies on the communication links. This, however, can be catered for by using long control cycles (i.e., relaxing IT needs) or using reliable communications channels (e.g., fiber optic).

From the planning perspective, given the EV adoption trends, traditional reinforcements can be compared against the proposed control to then select the most beneficial approach in the long term, particularly considering the effects on EV users.

\section{APPENDIX}

This appendix illustrates the actual operation of EV charg- 


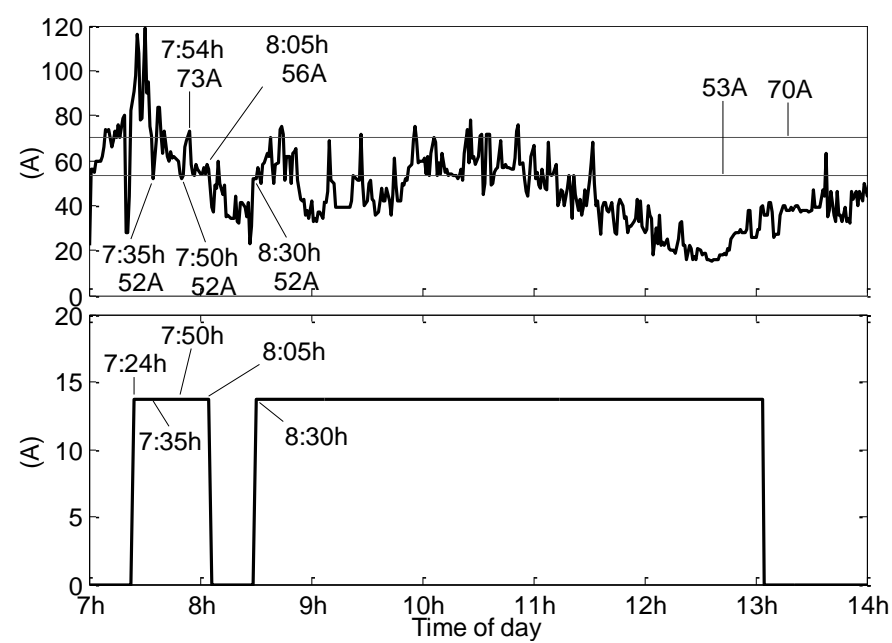

Fig. 18. Actual EV charging operation of the trialed control algorithm

ing points considering the architecture adopted by the 'My Electric Avenue' project [18] and presented in Fig. 6. Given that various adaptations of the control algorithm are tested progressively within the trial, here it is presented one that only manages the currents through the head of the feeder. Despite the simplified approach used in the trialed algorithm, previously discussed aspects such as the control cycle, thermal limits, security margin, and time-based ranking are adopted.

Here, the PLC at the substation receives every minute the average phase current in the last $10 \mathrm{~min}$. Within each 15 -min control cycle the algorithm checks whether the phase current exceeded the thermal limit. It also checks whether the phase current has constantly exceeded a specified security margin. If both excesses exist a signal to disconnect all charging points is triggered. A time-based ranking is used to reconnect each charging point every $25 \mathrm{~min}$ when below the margin.

Fig. 18 shows the EV charging operation for one of the phases of a feeder with a total of 57 customers, $670 \mathrm{~m}$ and 10 EVs in the North East of England. The control cycle starts at 0:05h. The thermal limit and security margin are $70 \mathrm{~A}$ and $17 \mathrm{~A}$, respectively. It can be seen that, although a high current occurs at around 7:30h, the current at 7:35h (control cycle) is $52 \mathrm{~A}$ (below the margin) thus not triggering disconnections. However, at 7:54h the current exceeds $70 \mathrm{~A}$ and remains above $53 \mathrm{~A}$ until 8:05h, resulting in the disconnection of charging points. The first EV (lowest charging time so far) is reconnected at 8:30h given that the current is $52 \mathrm{~A}$.

The above demonstrates that the architecture adopted in the trial and in this work is suitable for managing EV charging points. Moreover, it highlights that the proposed control algorithm as well as adaptations of it can be used in practice.

\section{X.ACKNOWLEDGEMENT}

The authors would like to thank Electricity North West Limited (ENWL), UK, for providing the LV network data.

\section{REFERENCES}

[1] Committee on Climate Change, "Reducing the UK's carbon footprint and managing competitiveness risks," Apr. 2013.

[2] L. Pieltain Fernández, T. G. S. Román, R. Cossent, C. M. Domingo, and P. Frías, "Assessment of the impact of plug-in electric vehicles on distribution networks," IEEE Trans. Pow. Syst., vol. 26, no. 1, pp. 206213, Feb. 2011.
[3] P. Richardson, M. Moran, A. Maitra, J. Taylor, and A. Keane, "Impact of electric vehicle charging on residential distribution networks: An Irish demonstration initiative," in CIRED, Stockholm, 2013.

[4] P. Richardson, D. Flynn, and A. Keane, "Optimal charging of electric vehicles in low-voltage distribution systems," IEEE Trans. Pow. Syst., vol. 27, no. 1, pp. 268-279, Feb. 2012.

[5] X. Luo and K. W. Chan, "Real-time scheduling of electric vehicles charging in low-voltage residential distribution systems to minimise power losses and improve voltage profile," IET Gener. Transm. Distrib, vol. 8, no. 3, pp. 516-529, Mar. 2014.

[6] J. Hu, S. You, M. Lind, and J. Østergaard, "Coordinated charging of electric vehicles for congestion prevention in the distribution grid," IEEE Trans. Smart Grid, vol. 5, no. 2, pp. 703-711, Mar. 2014.

[7] I. Sharma, C. Canizares, and K. Bhattacharya, "Smart charging of PEVs penetrating into residential distribution systems," IEEE Trans. Smart Grid, vol. 5, no. 3, pp. 1196-1209, May 2014.

[8] K. Clement-Nyns, E. Haesen, and J. Driesen, "The impact of charging plug-in hybrid electric vehicles on a residential distribution grid," IEEE Trans. Pow. Syst., vol. 25, no. 1, pp. 371-380, Feb. 2010.

[9] Z. Peng, Q. Kejun, Z. Chengke, B. G. Stewart, and D. M. Hepburn, "A methodology for optimization of power systems demand due to electric vehicle charging load," IEEE Trans. Pow. Syst., vol. 27, no. 3, pp. 16281636, Aug. 2012.

[10] E. Sortomme, M. M. Hindi, S. D. J. MacPherson, and S. S. Venkata, "Coordinated charging of plug-in hybrid electric vehicles to minimize distribution system losses," IEEE Trans. Smart Grid, vol. 2, no. 1, pp. 198-205, Mar. 2011.

[11] L. Hua, J. Wang, and C. Zhou, "Adaptive electric vehicle charging coordination on distribution network," IEEE Trans. Smart Grid, vol. 5, no. 6, pp. 2666-2675, Nov. 2014.

[12] J. de Hoog, T. Alpcan, M. Brazil, D. A. Thomas, and I. Mareels, "Optimal charging of electric vehicles taking distribution network constraints into account," IEEE Trans. Pow. Syst., vol. 30, no. 1, pp. 365-375, Jan. 2014.

[13] J. A. P. Lopes, F. J. Soares, and P. M. R. Almeida, "Integration of electric vehicles in the electric power system," Proceedings of the IEEE, vol. 99, no. 1, pp. 168-183, Jan. 2011

[14] Frontier Economics, "How to deliver smarter grids in GB," April 2011.

[15] A. Navarro, L. F. Ochoa, and D. Randles, "Monte Carlo-based assessment of PV Impacts on real UK low voltage networks," in IEEE PES General Meeting, 2013, pp. 1-5.

[16] S. Shengnan, M. Pipattanasomporn, and S. Rahman, "Grid integration of electric vehicles and demand response with customer choice," IEEE Trans. Smart Grid, vol. 3, no. 1, pp. 543-550, Mar. 2012.

[17] John Sean Sinclair, "Power Demand Management on a Low Voltage Network with a Plurality of Intelligent Sockets," GB Patent PCT/GB2012/050702, 2014.

[18] My Electric Avenue. Available: http://myelectricavenue.info/

[19] A. Navarro-Espinoza and L. F. Ochoa, "Report for Deliverable 3.6," The University of Manchester, LVNS Project, www.enwl.co.uk/lvns, 2014.

[20] EPRI. Open Distribution System Simulator, 2012.

[21] ELEXON, "Load Profiles and their use in Electricity Settlement," 2013.

[22] I. Richardson, M. Thomson, D. Infield, and C. Clifford, "Domestic electricity use: A high-resolution energy demand model," Energy and Buildings, vol. 42, no. 10, pp. 1878-1887, Oct. 2010.

[23] Office of National Statistics, "Families and Households, 2013," Statistical Bulletin, Oct. 2013.

[24] Nissan LEAF. Available: http://www.nissan.co.uk/

[25] IEC 60076-2 Ed. 2.0, "Power transformers - Part 2: Temperature rise," ed, 1993.

[26] IEEE Std C57.91-1995(R2004), "IEEE guide for loading mineral-oilimmersed transformers," ed, 2004

[27] Department of Trade and Industry, "The Electricity Safety, Quality and Continuity Regulations 2002," Oct. 2002.

[28] Ramon Vilanova and Antonio Visioli, PID Control in the Third Millennium Lessons Learned and New Approaches. Springer-Verlag London Limited, 2012.

[29] Cooper Bussmann. (2015, May). BS88:5 'J' - Type Feeder Pillar Fuse Link - SJ31-6. Available: http://www1.cooperbussmann.com/ pdf/3419dcb1-a607-4b21-bc4b-5a6048397e0e.pdf

[30] S. W. Alnaser and L. F. Ochoa, "Advanced network management systems: A risk-based AC OPF approach," IEEE Trans. Pow. Syst., vol. 30, no. 1, pp. 409-418, Jan. 2015.

[31] J. Bisschop and M. Roelofs, "AIMMS - The User's Guide," Paragon Decision Technology, 2006 